\title{
Galileo or for whom the bell tolls
}

\author{
K. Legat ${ }^{1}$ and B. Hofmann-Wellenhof ${ }^{1,2}$ \\ ${ }^{1}$ Tele Consult Hofmann-Wellenhof and Partner OEG, Austria \\ ${ }^{2}$ Graz University of Technology, Austria
}

(Received November 10, 1999; Revised August 2, 2000; Accepted August 3, 2000)

\begin{abstract}
Satellite-based navigation rapidly evolved into an efficient tool extensively used in a wide variety of civilian applications covering numerous modes of transportation, communication, administration, geodesy, agriculture, and many others. The current systems globally available are the US Global Positioning System (GPS) and the conceptually very similar Russian Global Navigation Satellite System (GLONASS). Considering the worldwide applications, GPS clearly predominates over GLONASS. However, GPS and GLONASS are mainly under military control of single nations and, also critical, do not fulfill certain performance requirements of the civil users, especially in terms of safety-critical applications. Thus, augmentations to the current systems and even completely new systems are under investigation. These are usually summarized under the abbreviation Global Navigation Satellite Systems (GNSSs). The various types of GNSS are described where emphasis is put on the future US and European contributions to the second-generation GNSS, i.e., the modernized GPS and the definition of the new European Galileo system. These two systems may be characterized as "compatible competitors" - thus, one might ask for whom the bell tolls.
\end{abstract}

\section{Introduction}

\subsection{GPS and GLONASS}

For the characteristics of GPS and GLONASS, the reader is referred to textbooks such as Hofmann-Wellenhof et al. (1997) or Feairheller et al. (1996). Subsequently, only the main shortcomings of the current systems are outlined which may be interpreted as the motivation for the development of the future GNSS. In addition, some of the most stringent civilian performance requirements are stated.

\subsection{Shortcomings of the current systems}

The main drawbacks of the current satellite-based navigation systems related to civilian applications are:

- GPS and GLONASS are owned and controlled by (military) institutions of single nations. Especially in case of political crises, additional restrictions may arise for civil users.

- Since the beginning of May 2000, the accuracy of GPS is no longer intentionally degraded for nonmilitary users by Selective Availability (SA). However, according to White House statements, the USA have found an alternative method to ensure the usability of GPS for military operations, cf. The White House (2000). This new technique is known as Selective Denial (SD) and allows to completely deny all GPS signals to civil users within a certain region by installing local jamming transmitters. Thus, SD may be even more critical to the civil user community than its predecessor SA.

Copy right $(\mathrm{C})$ The Society of Geomagnetism and Earth, Planetary and Space Science (SGEPSS); The Seismological Society of Japan; The Volcanological Society of Japan; The Geodetic Society of Japan; The Japanese Society for Planetary Sciences.
- The real-time non-augmented positional accuracy of both, GPS (even without SA) and GLONASS, is insufficient for many safety-critical applications.

- For certain ranges of application, the integrity of the systems does not meet the performance requirements of civil users. Aircraft precision approach and landing procedures as defined by the International Civil Aviation Organization (ICAO) are very typical examples for such applications.

- The availability of the current systems is insufficient for many safety-critical applications.

- With respect to continuity of service, there exist no or only limited guaranties by the responsible US and Russian authorities.

- Presently, the status of the space segment of GLONASS is critical making the system unusable for most civilian applications. At the beginning of July 2000, only ten "healthy" satellites of the nominal number of 21 active plus three spare satellites were in orbit. Most of these satellites have already exceeded their planned operational period. Without new launches in the near future, GLONASS might become unusable soon.

- The question of liability in case of an accident caused by a malfunction of the navigation system is not resolved satisfactorily for the current systems.

Considering these drawbacks, the performance of GPS and GLONASS appears unacceptable for a wide variety of civilian applications. For this reason, augmentations to the current systems or even completely new systems that offer a performance in accordance with the requirements of the civil 
Table 1. Accuracy requirements for precision approach and landing procedures.

\begin{tabular}{lccc}
\hline & & \multicolumn{2}{c}{ Accuracy $[\mathrm{m}](95 \%)$} \\
\cline { 3 - 4 } Procedure & Category & Horizontal & Vertical \\
\hline Precision & I & 18.2 & $4.4-7.7$ \\
approach & II & 6.5 & 1.7 \\
and landing & III & 4.1 & 0.6 \\
\hline
\end{tabular}

users are developed. However, one has to remember that GPS and GLONASS were primarily designed and developed as military systems.

\subsection{Civilian performance requirements}

In order to meet the maximum possible range of civilian performance requirements, future systems should be developed according to the needs of the most demanding users. With respect to navigation, the most challenging demands concerning accuracy, integrity, availability, and continuity arise from civil aviation. Giving an example of the high demands related to certain safety-critical applications, Table 1 shows the real-time accuracy requirements related to the different categories of precision approach and landing procedures as defined by ICAO. The situation for the other performance parameters is analogous.

These high requirements cannot be met by the current systems without integrating some appropriate augmentations. Even if the vertical accuracy of category III may be obtained by means of sophisticated differential positioning techniques, the ensemble of all performance parameters requires "some more activity".

\section{GNSS}

The development of GNSS is a two-step approach covering two distinct generations of systems: The first generation (GNSS-1) consists of augmentations to GPS and GLONASS. The second generation (GNSS-2) should finally evolve into a completely new system under (possibly international) civil control meeting almost all civilian performance requirements. The two generations of systems are described in some more detail, emphasizing primarily GNSS-2.

\subsection{GNSS-1}

The first-generation GNSS comprises two different components: (1) the Satellite Based Augmentation Systems (SBASs) which are regional systems of continental scale, and (2) the Ground Based Augmentation Systems (GBASs) operating on a local basis. An appropriate integration of these two types of systems enables a performance in compliance with the most challenging requirements of civil aviation.

\subsubsection{SBASs}

General Remarks The SBASs are regional augmentation systems with respect to GPS (and possibly GLONASS). These systems include a space segment of several geostationary satellites (GEOs) covering the operational area and a corresponding control segment in order to support aviation users during all phases of flight including non-precision approaches and probably precision approach category I. The SBASs offer three types of services:
- Ranging to the GEOs to improve availability and continuity of service.

- Determination and transmission of integrity information referring to the GPS (and GLONASS) satellites and the GEOs.

- Determination and transmission of Wide Area Differential (WAD) corrections for the GPS (and GLONASS) satellites and the GEOs to improve the positional accuracy.

The ranging capability is realized via the GEOs by transmitting a GPS like signal on the L1 carrier frequency. The integrity information and differential corrections are modulated onto this carrier similarly to the GPS data code but with a higher data rate.

Currently, three SBASs are under development worldwide. These are the US Wide Area Augmentation System (WAAS), the Japanese Multi-function Transportation Satellite Augmentation System (MSAS), and the European Geostationary Navigation Overlay Service (EGNOS). These systems are compatible and will achieve a globally seamless coverage with regional GNSS-1 services.

EGNOS Since EGNOS is meanwhile widely considered as an initial phase of the future European Galileo system, more details are given. The development of EGNOS is conducted by the European Tripartite Group (ETG) comprising the three European institutions European Commission (EC), European Space Agency (ESA), and Eurocontrol (European Organization for the Safety of Air Navigation) which are responsible for different duties in the development of EGNOS, cf. Benedicto et al. (1999).

The space segment of EGNOS is based on three GEOs, namely two Inmarsat-3 satellites referred to as AOR-E (Atlantic Ocean Region East) and IOR (Indian Ocean Region) and one ESA satellite denoted as Artemis. These satellites provide a threefold overlapping coverage of the central European region as well as of huge parts of the African continent.

The control segment of EGNOS will consist of four mission control centers situated in Germany, Great Britain, Italy, and Spain, two ground uplink stations per GEO satellite, and some 30-40 mainly European control stations denoted as RIMSs (Ranging and Integrity Monitoring Stations). This large number of control stations is required to determine the impact of the ionosphere on the satellite signals since only single-frequency data are available to the users. The whole control segment will be interconnected by a communication network based on terrestrial and satellite communication links.

The two Inmarsat-3 satellites are already in orbit, Artemis is due for launch during 2000. The Advanced Operational Capability (AOC) of EGNOS is scheduled for 2002. At present, trials of EGNOS are conducted to verify systematically that the service satisfies international regulations. The first trials focus on the aviation and maritime sectors, cf. European Commission (2000). Recently, no information is provided anymore concerning Full Operational Capability (FOC) of the system. The European Union (EU) has initiated a study investigating the possible integration of EGNOS into Galileo (denoted as INTEG). It is recommended to in- 
tegrate EGNOS at the ground and user level, i.e., to reuse the EGNOS ground infrastructure to the largest extent possible.

2.1.2 GBASs The GBASs (or Local Area Augementation Systems as they are called in the USA) represent the second component of GNSS-1. These systems, which will primarily be installed in the vicinity of airports, are expected to support aviation users during precision approach and landing procedures including category III, cf. Department of Defense and Department of Transportation (2000). The high requirements related to these procedures stipulate the inclusion of ground-based infrastructure to augment the basic GPS (and GLONASS) systems. Realistically, GBASs will also be required for GNSS-2 in order to fulfill the highest demands of civil aviation.

A single GBAS will comprise at least one reference station, one or more monitor stations, and several ground-based transmitters denoted as "Pseudolites" (pseudo-satellites) emitting a GPS like signal. To achieve the high accuracy demands of category III as outlined in Table 1, precise differential techniques based on phase observations are required.

\subsection{GNSS-2}

2.2.1 General remarks Similar to GNSS-1, GNSS-2 is expected to include several components. Essentially, the modernized GPS and the future European Galileo system will likely constitute the second-generation GNSS. Currently, GLONASS does not play a major role in these developments since-apart from plans to modernize the system with a new generation of modified satellites (GLONASS-M) - the future of GLONASS is fairly veiled. Nevertheless, negotiations with the EU indicate that Russia might contribute to the development of Galileo.

2.2.2 Modernized GPS Presently, an intense GPS modernization process is being prepared that will result in substantial benefits for the military and nonmilitary users. Improvements are related to the control segment, the space segment, and the signal structure.

Control Segment According to the Presidential Decision Directive (PDD) released in 1996, the maintenance of the Standard Positioning Service (SPS) for civilian, commercial, and scientific applications is guaranteed free of direct user fees. Furthermore, it was declared to turn off SA within a decade, i.e., by 2006 at the latest. (As already indicated, SA was stopped by the beginning of May, 2000.) Finally, the permanent Interagency GPS Executive Group (IGEB) was established. This board is commonly chaired by the Department of Defense (DoD) and the Department of Transportation (DoT) to balance the interests of the military and nonmilitary users.

Besides the PDD, the Accuracy Improvement Initiative (AII) was announced in 1996. This initiative integrates up to 14 new monitor stations of the US National Imagery and Mapping Agency (NIMA) to extend the original GPS tracking network with only five monitor stations. Based on the enhanced control segment, additional data concerning orbit, clocks, and health of the GPS satellites may be obtained. These data result in improved navigation messages and shorter navigation uploads up to three times per day and, thus, in a decreased age of data. In addition, the AII increases the integrity of the system because possible malfunctions of the satellites may be detected at an earlier stage.
Finally, the concept of Autonomous Navigation (Auto-Nav) is mentioned. This technique may retain a threedimensional satellite position accuracy of $\pm 16 \mathrm{~m}(50 \%)$ over 60 days without ground contact. Cross links which enable to transfer navigation information between the satellites are the point of the concept. Presently, the Master Control Station must upload navigation data to each satellite separately.

Space segment and signal structure The current space segment of GPS mainly consists of Block II and Block IIA satellites. In the future, these satellites will gradually be replaced by the new Block IIR and Block IIF satellites being capable of the aforementioned Auto-Nav facility. For the time scale beyond 2030, a new generation of satellites belonging to Block III is envisaged. Referring to Block IIR satellites, two were launched successfully, one was lost due to a booster failure, and 18 more of this type are to follow.

The current GPS modernization plan foresees to add new military signals with a different code modulation structure to some of the Block IIR and Block IIF satellites. These new military signals are based on the so-called M-code which will have a non-central signal structure. Additionally, the new GPS satellites will also offer new civil capabilities. In detail, the current schedule is as follows, cf. Montgomery (2000):

The last 12 Block IIR satellites to be launched will be provided with the military M-code on L1 and L2 with increased power and the existing carrier L2 will be modulated with the civil C/A code analogously to L1. The first of these satellites is scheduled for launch in 2003.

The first 12 Block IIF satellites will be provided with a third civil signal on a new carrier frequency denoted as L5 with a frequency of $1176.45 \mathrm{MHz}$. Also, the M-code will be added to the new carrier. Nominally, the Block IIF satellites have an operational period of at least 10 years and will come into operation starting in 2006 or later. Contrary to the original plans, no further Block IIF satellites will be launched but the constellation will be gradually replaced by the future Block III satellites.

A summary of the prospected GPS carrier frequency structure is given in Table 2 where $f_{0}$ represents the fundamental GPS frequency of $10.23 \mathrm{MHz}$.

The new signals offer great advantages for the civil GPS users. The L1/L5 ionosphere-free combination improves the elimination of the ionosphere whereas the L2/L5 widelane combination with a $5.8 \mathrm{~m}$ wavelength facilitates ambiguity resolution. Also, three-carrier ambiguity resolution techniques are under investigation offering (almost) instantaneous ambiguity resolution.

\subsubsection{Galileo}

General remarks For several years, different political institutions within the EU have been investigating how Europe could enlarge its market share in the field of satellitebased navigation. In negotiations with the USA, Europe tried to become involved into the further development of GPS. However, the USA declared that GPS must remain under national control. Therefore, the EU decided to develop its own system leading to two projects, namely EGNOS and Galileo.

After Wlaka (1997), the European motivation is threefold: The development of a European system (1) results in a variety of benefits for the civil user community, (2) strengthens 
Table 2. Future GPS frequency spectrum.

\begin{tabular}{cl}
\hline Carrier & \multicolumn{1}{c}{ Remarks } \\
\hline $\mathrm{L} 1=154 f_{0}=1575.42 \mathrm{MHz}$ & Existing carrier \\
$\mathrm{L} 2=120 f_{0}=1227.60 \mathrm{MHz}$ & Existing carrier additionally modulated with C/A-code \\
$\mathrm{L} 3=135 f_{0}=1381.05 \mathrm{MHz}$ & Existing military signal (no public access) \\
$\mathrm{L} 5=115 f_{0}=1176.45 \mathrm{MHz}$ & New carrier for civil and military use \\
\hline
\end{tabular}

the European economy and sovereignty, and (3) enables the European industry to take a leading role in the emerging high technology market of satellite-based navigation.

In June 1999, the European Ministers of Transport agreed on the definition phase of Galileo based on previous work done by the European GNSS Forum. Galileo will be an open and global system under international civil control, fully compatible and interoperable with GPS (and maybe GLONASS) but independent of it. Three options for cooperation in the development of Galileo are under discussion: (1) Global development together with all other interested partners, (2) joint development together with one or more international partners such as the USA, Russia or Japan, and (3) independent development of a global system solely by the EU.

As stated above, the USA have declared the indispensable condition that GPS remains under US control. A cooperation is only possible if the EU accepts the SPS and the related signal structure as the international standard for GNSS. Russia proposed a joint development of GNSS-2. At the same time, the Russian authorities announced the intention to convert GLONASS into a system under civil control which could gradually evolve into Galileo. In this case, the development of Galileo could be accelerated by the advantage of Russian know-how and by using the allocated GLONASS frequency band. Japan is developing its own GNSS-1 component but does not express much interest in national contributions to GNSS-2. However, according to European Commission (1999), the Japanese show increasing interest in a joint development of Galileo although they have recently recognized GPS as the global standard for satellite navigation. Canada will actively cooperate with ESA in the development of the control and space segments of Galileo. Further, a number of other countries from all over the world have indicated interest in a cooperation with the EU.

System architecture In contrast to previous proposals by several European companies, Galileo will have a global system architecture. The main reason for this decision is that the EU could not find any international partners developing counterparts to a regional European system to guarantee globally seamless navigation services.

In the following paragraphs, the current state of the Galileo design is explained based on European Commission (2000). Since Galileo is still in its definition phase, changes to the proposed system architecture are likely to occur. The related ESA development initiative for the space and ground segments of Galileo is denoted as GalileoSat.

Control segment. Some components of the control seg-
Table 3. Galileo frequency spectrum reserved at WRC-2000.

\begin{tabular}{cc}
\hline Frequency band $[\mathrm{MHz}]$ & Remarks \\
\hline $1190-1215$ & E5, L-band \\
$1260-1300$ & E6, L-band \\
$5010-5030$ & C1, C-band \\
\hline
\end{tabular}

ment of EGNOS will be reused for Galileo although additional ground stations are required outside Europe in order to achieve global coverage. The Galileo control segment will be interconnected by a communication network and will consist of two independent chains of almost autonomous operation. This concept enables internal integrity control and ensures high quality operation.

Space segment. Presently, two different options for the space segment of Galileo mainly based on Medium Earth Orbit satellites (MEOs) are under consideration. (The space segments of GPS and GLONASS also consist of MEOs.) The two constellation options under consideration are (1) a space segment consisting of 30 MEOs, and (2) a space segment consisting of 24 MEOs and eight GEOs. With respect to safety-critical applications, both options require additional augmentation by means of GBASs. Currently, the MEO-only option appears most promising for various reasons including the homogeneity of the service and the costs for satellite launch and replenishment.

Signal structure. With respect to the signal structure of Galileo, several important decisions have been taken at the last World Radio Conference (WRC) that took place at Istanbul, Turkey, in May and June 2000. The frequency bands reserved for Galileo are summarized in the Table 3, cf. Vorhies (2000):

Apart from these three frequency bands, three narrow band signals within the L-band have already been reserved for Galileo prior to WRC-2000. In contrast to EGNOS, all Galileo users will have access to at least two civil signals. This allows the users to estimate the ionospheric refraction. Therefore, no provision for ionospheric corrections on the signal is necessary. However, Galileo users with high safety requirements will need additional system integrity information as provided, e.g., by the SBASs.

Services and performance. According to European Commission (1999), Galileo will offer three different kinds of navigation services: (1) An Open Access Service (AOS) as the basic public service for the mass market-free of charge as long as the SPS is free of charge, (2) a Controlled Ac- 
Table 4. Preliminary performance parameters for an operation of autonomous Galileo.

\begin{tabular}{lc}
\hline \multicolumn{1}{c}{ Parameter } & Requirement \\
\hline Accuracy $(95 \%)$ & $4.0 \mathrm{~m}$ \\
Horizontal & $7.7 \mathrm{~m}$ \\
Vertical & $30 \mathrm{~ns}$ \\
Timing & \\
\hline Integrity & $6.10^{-7}$ per $150 \mathrm{~s}$ \\
Risk & $12 \mathrm{~m}$ \\
Time-to-alarm & $0.9-0.9997$ \\
Alarm limit (recommended) & $8.10^{-6}$ per $15 \mathrm{~s}$ \\
\hline Availability
\end{tabular}

cess Service (CAS1) with user fees designed for users that require a guaranteed service, possibly containing a liability cover, and (3) another Controlled Access Service (CAS2) for military use and safety-critical applications.

The basic civil navigation service of Galileo must provide at least the same performance as the modernized GPS based on the future Block IIF satellites. Registered users of the premium service will be backed by liability regulations limiting commercial risks in case of any system failure. The preliminary performance parameters for an autonomous operation of Galileo are contained in Table 4. The values listed mainly refer to the land applications, some exceptions are permitted over oceanic regions. The constellation of Galileo is designed to provide an optimized performance even at higher latitudes.

Communication function. In the future, more and more applications of navigation services will be integrated with communication services, e.g., in fleet management for transport purposes the central will have to know where the different vehicles are actually located. Thus, two types of communication services are envisaged for Galileo: On the one hand, there are plans for a service with a very low bit rate (less than $50 \mathrm{bps}$ ) embedded in the navigation signal, and on the other hand, a dedicated communications payload could be installed onboard the satellites possibly providing a two-way communication service with data rates up to $100 \mathrm{kbps}$.

Financial aspects The prospected cost of the entire Galileo program primarily depends on two factors, namely the number of satellites and the inclusion of the above mentioned CASs. The maximum total cost of the system amounts to about 3 billion Euros (approximately US\$ 3 billion). The annual cost for the operation of the system is expected to be 140-205 million Euros. A four-point strategy has been proposed for financing Galileo, cf. Galileo Task Force (1999):

- Substantial financing at the European level through the EU budget, notably through the Trans-European Networks and research and development programs, and through ESA.

- Acquisition of additional funds through involvement of other EU member states' agencies or institutions.

- Mobilization of additional financing or achievement of savings through international cooperation with third countries such as Russia, Japan or Canada.

- Complementary financing by developing a PublicPrivate-Partnership (PPP).

Moreover, the EU considers to apply the "user-paysprinciple" within Galileo which requires the introduction of appropriate revenue streams. Possible revenue streams identified include: a general levy on all GNSS receivers (including also GPS and GLONASS receivers), fees related to the two CASs, and charges for the integration of the navigation services with an additional communication function.

Schedule and risks The development of Galileo will be carried out in four phases: (1) The definition/design phase which was entered in June 1999 and shall be finished by the end of 2000 or in early 2001, (2) the development phase where the infrastructure is developed and validated, (3) the deployment phase where the complete system is put into place, and (4) the operation phase where the services are offered and the system is maintained and developed.

The final decision on continuing the development of Galileo will be taken after the completion of the first phase. If the EU decides to realize the system, a test constellation of 3-5 MEOs will be introduced around 2003; the start of signal transmission is scheduled for 2005, and the whole system could be completed by 2008 .

Possible risks identified in the Galileo program by the responsible Task Force primarily refer to the schedule of the system. Major risks of concern are that the development program as explained above may last long. Thus, the current opportunity may be lost to introduce Galileo before the GPS modernization is complete. Furthermore, significant, competing technologies might arise during the development phase of Galileo. These risks become even more critical if the possibly accelerated modernization of GPS is considered.

\subsubsection{GPS and Galileo-competitors or partners?}

Considering the various aspects explained above, there is certainly a twofold relationship between GPS and Galileo. On the one hand, the two systems are competitors concerning the time schedules of their modernization and development, respectively. The USA have clearly indicated their intention to promote GPS as the world standard for GNSS. Under the aspect of its evolving Common Foreign and Security Policy (CFSP), the EU wants to avoid the dependency on a foreign military controlled navigation system. Also, the Europeans have already presented their Galileo plans to NATO, cf. Divis (1999b).

On the other hand, Galileo will be compatible and interoperable with GPS which will involve huge benefits for the USA, the EU and for many users all over the world. The two independent systems could be used either alternately or in combination resulting in a far better overall performance. However, if Galileo and GPS were incompatible, the USA would - despite of any budgetary reservationscertainly further accelerate the GPS modernization in order to keep up their national interests related to this system.

Until today, the US reaction to the European Galileo pro- 
Table 5. Comparison of the modernized GPS and Galileo.

\begin{tabular}{lcc}
\hline & Modernized GPS & Galileo \\
\hline Technical features & $24+0$ & $30+0(24+8)$ \\
Satellites: MEOs + GEOs & 6 & 3 \\
Orbital planes & $55^{\circ}$ & $54^{\circ}$ \\
Inclination & $20200 \mathrm{~km}$ & $23000 \mathrm{~km}$ \\
Altitude & signals (L1, L2, L5) & 3 wide-band signals (E5, E6, C1) \\
Signal structure (civil) & SPS & OAS, CASs \\
Civil navigation services & & Civil \\
\hline Further features & Military & Partly (CASs) \\
Control & No & Partly (CASs) \\
Liability cover & No & Bi-directional \\
User fees & No & 2008 \\
Communication function & $?$ & \\
Completion of development & & \\
\hline
\end{tabular}

posal was rather cautious. The only item causing major discussions was the financing strategy presented in the communication of the EC released in February 1999. As indicated, the general levy on GNSS receivers would mean that the US industry helps indirectly financing Galileo, cf. Divis (1999a).

In summary, Table 5 lists some of the main features of the modernized GPS and Galileo as they are currently defined.

\section{Conclusions}

The present satellite-based navigation systems GPS and GLONASS do not meet the performance requirements associated with a wide variety of civilian applications. Thus, augmentations to the current systems and even completely new systems are under investigation. These are usually summarized under the abbreviation GNSS.

Today, the most important of these upcoming systems are the modernized GPS and the future European Galileo system. In the same way as GPS evolved into a very important element of the US economy, the European contribution in the development of GNSS will become a key factor for the European industry in the next century. In detail, Galileo is expected to induce an additional macro-economic benefit of 90 billion Euros in the application industry over the period 2005-2025. Furthermore, Galileo will probably create more than 100000 new, high technology jobs all over Europe, cf. Tytgat (1999).

Taking into account these far-reaching benefits related to satellite-based navigation, it appears obvious that the modernized GPS and Galileo will be competitors. Currently, the USA more or less own the monopoly in this field. The Europeans want to compete with the introduction of Galileo. But the ambitious Galileo plans must be realized before the GPS modernization is complete. Then one might ask: GPS or Galileo? Or: for whom the bell tolls.

Acknowledgments. Some paragraphs of this paper are closely related to Lichtenegger (1999) who gives an excellent summary of Europe's way to GNSS.

\section{References}

Benedicto, J., P. Michel, J. Ventura-Traveset, and T. Maier, EGNOSEuropean Geostationary Navigation Overlay Service, basic technical background, Proceedings of the Workshop on DGPS and DGLONASS Techniques and Application on Safety in CEI Area, Trieste Italy, 912 March, Warsaw University of Technology, Institute of Geodesy and Geodetic Astronomy, No. 3(44), 1999.

Department of Defense and Department of Transportation, 1999 Federal Radionavigation Plan, U.S. National Technical Information Service, Springfield, Virginia 22161, DOT-VNTSC-RSPA-98-1/DOD-4650.5, 2000.

Divis, D. A., Galileo: Competition or complement for GPS, GPS World, 10(4), 14-20, 1999a.

Divis, D. A., More on Galileo: EU takes its plan to NATO, Western European Union, GPS World, 10(5), 14-19, 1999 b.

European Commission, Galileo: Involving Europe in a New Generation of Satellite Navigation Services, Communication, Brussels, 10 February, 1999 .

European Commission, Galileo Definition Phase-Initial Results, June 7, 2000, Directorate-General for Energy and Transport, Directorate BTranseuropean Networks Energy and Transport Satellite Navigation Systems (Galileo), 2000.

Feairheller, S., J. Purvis, and R. Clark, The Russian GLONASS system, in Understanding GPS Principles and Applications, edited by E. D. Kaplan, pp. 439-465, Artech House, Boston London, 1996.

Galileo Task Force, Galileo Task Force on Public Private Partnership, Chairman's Report, Report to Commissioner Neil Kinnock, Brussels, 4 June, 1999.

Hofmann-Wellenhof, B., H. Lichtenegger, and J. Collins, GPS theory and practice, 4th edition, 389 pp., Springer, Wien New York, 1997.

Lichtenegger, H., Europe on the way to GNSS, International Symposium "Modern Information and GPS Technology-Aspects and Implications of Their Application", Sofia, 11-12 November, 1999.

Montgomery, H. (ed.), GPS III and Modernization, Newsletter of the Institute of Navigation, 10(1), 7, 2000.

Tytgat, L., Galileo-Global Satellite Navigation Services for Europe, Brochure of the European Commission Directorate-General VII Transport, Brussels, 1999.

Vorhies, J., WRC Results-GPS, www . igeb.gov, 2000.

The White House, Statement by the President regarding the United States decision to stop degrading Global Positioning System accuracy, www. pub. whitehouse.gov/uri-res/I2R?urn:pdi : // oma . eop.gov.us/2000/5/2/7. text. 1, 2000.

Wlaka, M., The European contribution to GNSS 2, a technical concept, GNSS 97, Munich 21-24 April, 1997.

K. Legat (e-mail: legat@tu-graz.ac.at) and B. Hofmann-Wellenhof 